\title{
Pitfalls in the Interpretation of Publications about Endoscopic Submucosal Dissection of Early Gastric Cancer with Undifferentiated-Type Histology
}

\author{
Chang Seok Bang and Gwang Ho Baik \\ Department of Internal Medicine, Hallym University College of Medicine, Chuncheon Sacred Heart Hospital, Chuncheon, Korea
}

Endoscopic submucosal dissection (ESD) is a standard treatment for patients with gastrointestinal neoplasms with a negligible risk of lymph node metastasis. ESD enables en bloc resection of gastrointestinal neoplasms and organ preservation, thereby, avoiding surgical treatment. Although small $(<2 \mathrm{~cm})$ intramucosal early gastric cancer with undifferentiated-type histology (EGC-UH) without ulceration is included in the expanded criteria for ESD, controversies remain due to different biology and characteristics compared to EGC with differentiated-type histology. The authors previously presented studies about the technical feasibility of ESD for these lesions using a meta-analysis and retrospective multicenter analysis. However, many pitfalls were identified in the interpretation of studies analyzing histologic discrepancy, mixed-type histology, criteria-based analysis of therapeutic outcomes, interpretation of curative resection, and long-term clinical outcomes. In this review, the authors discuss pitfalls in the interpretation of publications on ESD for EGC-UH.

Clin Endosc 2019;52:30-35

Key Words: Carcinoma; Endoscopic submucosal dissection; Undifferentiated

\section{INTRODUCTION}

Endoscopic submucosal dissection (ESD) is a primary treatment for superficial neoplasms of the gastrointestinal tract with negligible risk of lymph node metastasis (LNM). ${ }^{1}$ This enables en bloc resection of the neoplasm and organ preservation, thereby avoiding invasive surgery. After histologic confirmation, endoscopic resection of a lesion satisfying the indication for ESD is performed. Pathologic confirmation of the resected specimen determines whether curative resection was achieved (satisfying post-ESD criteria). Curative resec-

Received: September 9, 2018 Revised: October 30, 2018

Accepted: November 2, 2018

Correspondence: Chang Seok Bang

Department of Internal Medicine, Hallym University College of Medicine, Chuncheon Sacred Heart Hospital, 77 Sakju-ro, Chuncheon 24253, Korea Tel: +82-33-240-5821, Fax: +82-33-241-8064, E-mail: csbang@hallym.ac.kr ORCID: https://orcid.org/0000-0003-4908-5431

(c) This is an Open Access article distributed under the terms of the Creative Commons Attribution Non-Commercial License (http://creativecommons.org/ licenses/by-nc/3.0) which permits unrestricted non-commercial use, distribution, and reproduction in any medium, provided the original work is properly cited. tion implies a favorable long-term outcome. For early gastric cancer (EGC), the indication for ESD has been expanded with advances in endoscopic skills. ${ }^{2,3}$ However, EGC with undifferentiated-type histology (EGC-UH), including poorly-differentiated adenocarcinoma (PDC) or signet ring cell carcinoma (SRC), has distinctive growth patterns, and ESD for these lesions is still controversial, especially because the defining indications are unclear. ${ }^{4}$ Although small $(<2 \mathrm{~cm})$ intramucosal EGC-UH without ulceration is included in the expanded criteria, controversies remain due to different biology and characteristics compared to EGC with differentiated-type histology (EGC-DH). ${ }^{5-8}$ The authors previously presented studies about the technical feasibility of ESD for these lesions using a meta-analysis and retrospective multicenter analysis. ${ }^{4,5}$ However, many pitfalls were detected in the interpretation of some topics in these studies. In this review, the authors discuss pitfalls in the interpretation of publications about ESD for EGCUH. 


\section{HISTOLOGY}

\section{Histologic discrepancy}

Precise histologic diagnosis is important before ESD, especially for EGC-UH. EGC-DH on biopsy before ESD can be changed to EGC-UH after ESD, leading to a change in overall treatment strategy. Current indications for ESD are focused on tubular adenocarcinoma and divided by the differentiation of EGC based on a Japanese classification that categorizes gastric adenocarcinoma into differentiated and undifferentiated types. ${ }^{9,10}$ The differentiated group in the Japanese classification for ESD refers to well- or moderately-differentiated tubular adenocarcinoma or papillary adenocarcinoma, and the undifferentiated group refers to PDC, SRC, or mucinous adenocarcinoma. ${ }^{9,10}$ Among the 4 predominant histologic types in the World Health Organization (WHO) classification, papillary adenocarcinoma is usually categorized into the differentiated group and SRC into the undifferentiated group; however, tubular adenocarcinoma is categorized according to differentiation and mucinous adenocarcinoma is classified according to predominant components in either the differentiated or undifferentiated group. $^{9-12}$

Endoscopists usually adopt indications based on the pathologic results of endoscopic biopsy before ESD. Therefore, there is a possibility of discrepancy between pre-ESD biopsy of the lesion and post-ESD biopsy of the resected specimen. In Korean real-practice data for gastric dysplasia, EGC, or advanced gastric cancer, the final pathology was upgraded in $15.9 \%$ of cases and downgraded in $6.9 \%$ after ESD or surgery, compared to that on the initial endoscopic biopsy. ${ }^{13}$ In a Korean-ESD multicenter study including only EGC-UH cases after ESD, 54.9\% showed pre- and post-ESD histologic discrepancy. ${ }^{5}$ Other studies also reported rates of histologic discrepancy of up to $84.7 \%$ in EGC-UH, compared to $16.3 \%$ to $53.7 \%$ for EGC-DH. ${ }^{14}$ The difference in discrepancy rates among studies can be explained by the difference in number of biopsy specimens, selection of biopsy sites in each study, and histologic heterogeneity. ${ }^{15}$ A previous study with histologic mapping showed that the zone of transition from differentiated to undifferentiated histology was frequently found at 1 or 2 peripheral sites of the lesion. ${ }^{15}$ Therefore, biopsy of peripheral sites may aid in accurate diagnosis. ${ }^{16}$ Inaccurate initial determination of differentiation leads to inappropriate application of the indications and occurs more frequently in EGC-UH than in EGC-DH. However, this factor was often not considered and the majority of studies on therapeutic outcomes of ESD for EGC-UH focused on post-ESD histology. ${ }^{4,5}$ Among 14 studies included in the previous meta-analysis, ${ }^{4}$ only 2 evaluated pre-ESD diagnosed cases of EGC-UH. ${ }^{17,18}$ EGC-UH showing differentiated histology on pre-ESD biopsy is associated with a lower curative resection rate than that for undifferentiated histology on pre-ESD biopsy, and inaccurate initial diagnosis could affect the therapeutic outcomes of ESD. ${ }^{15,16}$

\section{EGC with mixed-type histology}

EGC with mixed-type histology (EGC-MH, differentiated-type combined with undifferentiated-type) is another consideration. Because EGC-MH is histologically heterogeneous, diagnosis is challenging. It is only diagnosed after ESD or surgery as pure-type gastric cancer on initial biopsy must be ruled out. ${ }^{19}$ Currently, differentiated-type-predominant EGC mixed with an undifferentiated component is considered EGC-DH, whereas undifferentiated-type-predominant EGC mixed with a differentiated component is considered EGC-UH. ${ }^{20}$ However, EGC-MH is known to be associated with more submucosal invasion and higher risk of LNM or lymphovascular invasion than pure-type gastric cancer, irrespective of whether the mixed component is differentiated or undifferentiated, although the mechanism is unclear. ${ }^{19,21-26}$

With respect to therapeutic outcomes, retrospective analysis of surgical data showed that EGC-MH showed no LNM among lesions that met the present ESD criteria. ${ }^{27}$ Retrospective analysis of ESD data also showed that differentiated-type-predominant EGC mixed with an undifferentiated component showed no LNM or extra-gastric recurrence if the lesions met the present ESD criteria. ${ }^{28}$ However, another retrospective analysis of endoscopic resection data showed that differentiated-type-predominant EGC mixed with an undifferentiated component was a significant risk factor for non-curative resection regardless of tumor size. ${ }^{29}$

Papillary adenocarcinoma is usually defined as a tumor in which more than $50 \%$ of the involved area contains papillary structures. ${ }^{30}$ Although papillary adenocarcinoma is usually categorized into the differentiated group in the Japanese classification, ${ }^{10}$ papillary adenocarcinoma mixed with other differentiated-type EGC, or papillary adenocarcinoma mixed with undifferentiated-type EGC, is also classified according to the predominant component in the entire cancer. Surgical data on papillary adenocarcinoma in EGC showed an LNM rate of $17.9 \%$ among all lesions, and an LNM rate of $11.8 \%$ and lymphovascular invasion rate of $17.6 \%$ for lesions that met the current ESD criteria, although adoption of the same ESD criteria for differentiated tubular adenocarcinoma is unlikely. ${ }^{31}$ However, another study with surgical data on papillary adenocarcinoma in EGCs showed LNM rates comparable to those in differentiated tubular adenocarcinoma (1.5\%, 1.1\%, and $4.0 \%$ for mucosal EGCs and $9.45 \%, 11.9 \%$, and $17.6 \%$ for submucosal EGCs, in papillary EGC, differentiated tubular EGC, or EGC-UH, respectively), despite persistent aggressive features of higher lymphovascular invasion or submucosal 
invasion rates. ${ }^{32}$ Moreover, no LNM occurred in lesions that met the current ESD criteria for papillary adenocarcinoma. ${ }^{32}$ To explain this discrepancy between studies and between high lymphovascular invasion and low LNM rates, further research is needed.

Overall, simple discrimination into differentiated-type-predominant-, or undifferentiated-type-predominant mixed EGC might not reflect the feasibility of ESD for EGC-MH.

\section{PDC or SRC: a single category?}

Treatment of PDC and SRC as a single category (EGC$\mathrm{UH}$ ) is another consideration. Although included in the same category in the indications for ESD, they have different biological behaviors. PDC is associated with higher risk of LNM and SRC is associated with lower risk of LNM than that of other histologic types. ${ }^{33,34}$ With respect to the ESD procedure, vertical margin involvement was more frequent in PDC, and lateral margin involvement was more frequent in SRC, probably due to the subepithelial spreading subtype. ${ }^{5}$ Subepithelial spreading SRC is more prevalent than epithelial spreading type in cases with atrophy and intestinal metaplasia. ${ }^{16,35}$ Therefore, larger safety margins were recommended in ESD for SRC with surrounding mucosa exhibiting atrophy or/and intestinal metaplasia. ${ }^{16,35}$ The rate of lymphovascular invasion was higher in ESD for PDC than for SRC. ${ }^{5}$ However, therapeutic outcomes are similar between PDC and SRC if lesions meet the current ESD criteria. ${ }^{5}$ Moreover, recent surgical data showed no LNM in association with Tla tumors with PDC $<2 \mathrm{~cm}$ without lymphovascular invasion and Tla tumors with SRC $<1 \mathrm{~cm}$ without lymphovascular invasion, suggesting that more strict and separate ESD indications should be applied according to the histology. ${ }^{36}$

A mixture of PDC and SRC is another consideration. In a retrospective analysis of surgical data, a mixture of PDC and SRC showed an LNM rate of 6.3\%, which is higher than in pure-type PDC or SRC. ${ }^{36}$ Moreover, an LNM rate of 5.3\% was observed when this mixture of PDC and SRC met the current ESD criteria. ${ }^{36}$ Retrospective analysis of ESD data also showed that a mixture of PDC and SRC showed lower curative resection rates than pure SRC ( $77.7 \%$ vs. $93.8 \%)$ and was associated with non-curative resection in a multivariate analysis (odds ratio, 2.746; 95\% confidence interval, 1.162-6.485), in addition to more submucosal invasion and positive vertical margins. ${ }^{37}$

Considering the different origins and spreading patterns of PDC and SRC, separate and more strict indications might be required, although current guidelines recommend different treatment strategies only for EGC-DH and EGC-UH. ${ }^{5}$

\section{Gastric carcinoma with lymphoid stroma}

Gastric carcinoma with lymphoid stroma (GCLS), known as gastric lymphoepithelioma-like carcinoma or medullary carcinoma, is a rare histologic subtype of gastric cancer characterized by poorly differentiated tubular structures mixed with prominent lymphoid infiltration. ${ }^{38}$ The incidence of GCLS is $1 \%-4 \%$ of all gastric cancers. More than $80 \%$ of cases are associated with Epstein-Barr virus infection and 7\%-39\% of cases have microsatellite instability-high status. ${ }^{39-44}$ GCLS is known to have a favorable prognosis with a low LNM rate, although it is usually considered gastric cancer with undifferentiated-type histology. ${ }^{45,46}$ Increased tumor-infiltrating lymphocytes, which reflect a host immune response to tumor cells, are known to be associated with reduced metastasis and improved survival. ${ }^{46,47}$ Despite paucity of data, the feasibility of ESD for early GCLS has been evaluated and retrospective analysis of 52 surgically resected and 18 endoscopically resected early GCLSs showed lower LNM than 1,626 surgically resected EGCs other than GCLS (4.0\% vs. $19.4 \%){ }^{48}$ Recurrence was not detected in the 10 early GCLSs treated only with ESD during a mean follow-up of 37.2 months. ${ }^{48}$ The unique feature was that submucosal invasion was more frequent and deeper in early GCLSs than in surgically resected EGCs other than GCLS; however, the LNM rate was lower, even in GCLS invading the SM3 layer. ${ }^{48}$ Determination of the feasibility of ESD for GCLS according to the current indications requires more data.

Considering all data, subdivision by indication and validation is still necessary to lessen the limitations on ESD according to the histology of EGC.

\section{INDICATION OR CRITERIA?}

The current indication for ESD is based on retrospective analyses of surgical data for gastric cancer with negligible risk of LNM. Relevant local factors include size, depth of invasion, ulceration, and differentiation of lesions. The accurate prediction of pre-ESD indications is confirmed after ESD, based on whether the post-ESD criteria were satisfied. However, discrepancy between pre-ESD indications and post-ESD criteria was observed in $36.7 \%$ of all ESD cases of EGC-UH (out-of-indication rate) in a Korean multicenter study. ${ }^{5}$ Preand post-ESD histologic discrepancy as noted above is a factor in the out-of-indication rate leading to non-curative resection. Another factor is local characteristics of the lesion including size, depth of invasion, and ulceration. Lateral margin prediction is known to be low in ESD for SRC and deep margin prediction is low in ESD for PDC. 5.49 Owing to the unpredictability of the depth of invasion and lateral margin of EGC-UH, studies including all cases of resected EGC-UH regardless of the indication or criteria reflect actual experience. ${ }^{5}$ 
The reasons for non-curative resection in a Korean multicenter study including all cases of resected EGC-UH were multifactorial. Inaccurate prediction of the lateral margin (size $>2 \mathrm{~cm}$ ) was the most frequent reason (71.4\%) and inaccurate prediction of the deep margin (submucosal invasion) was the second most frequent reason (32\%). ${ }^{5}$ Positive lymphovascular invasion was an additional reason for non-curative resection $(14.9 \%))^{5}$ Therefore, biopsy of peripheral sites of lesions for accurate diagnosis of EGC-UH and use of larger safety margins in ESD for SRC with surrounding mucosa exhibiting atrophy or/and intestinal metaplasia can be considered to reduce non-curative resection of EGC-UH. ${ }^{16}$

Therapeutic outcomes of ESD for EGC-UH were not significantly different between the previous meta-analysis and a retrospective Korean multicenter study, except for curative resection (Table 1). ${ }^{4,5}$ Immediate therapeutic outcomes including en bloc resection and complete resection rate were similar in both studies, indicating technical feasibility of ESD for EGC-UH. However, curative resection rates were significantly different between the two studies. Curative resection indicates favorable long-term outcomes and non-curative resection indicates the risk of local recurrence or lymph node recurrence at a later stage, unless additional surgical or endoscopic resection is performed. ${ }^{1,737}$ The curative resection rate of ESD for EGC-UH ranged from $63.9 \%$ to $85.4 \%$ and is known to be lower in EGC-UH than in EGC-DH. ${ }^{37,50}$ The pooled curative resection rate of EGC-UH in a meta-analysis was $61.4 \% .{ }^{4}$ However, the curative resection rate in a Korean multicenter study was $36.4 \%$ regardless of the indication or criteria (Table 1). ${ }^{5}$ Some studies in a meta-analysis included
post-ESD diagnosed lesions limited to expanded criteria and presented the therapeutic outcomes. ${ }^{17,51,52}$ However, this might exaggerate the therapeutic outcomes of ESD for EGC-UH, because of frequent histologic discrepancy and out-of-indication rates for EGC-UH. ${ }^{4}$ Although the therapeutic outcomes of ESD for lesions that met the ESD criteria were more favorable than those for lesions beyond the ESD criteria, it is not always possible to apply accurate ESD indications. There has been consideration about use of post-ESD criteria in the enrollment of study participants, but little consideration about pre-ESD indications. ${ }^{4}$ Therefore, studies including all cases of resected EGC-UH regardless of the indication or criteria reflects actual experience.

\section{CURATIVE RESECTION AND LONG- TERM CLINICAL OUTCOMES}

Although curative resection results in favorable long-term outcomes (local recurrence, LNM, distant metastasis, survival) and non-curative resection indicates the risk of local recurrence or lymph node recurrence at a later stage, ${ }^{1,7,37}$ lower curative resection rates with favorable long-term outcomes are frequent in EGC-UH. A retrospective nationwide registry of endoscopic resection for EGC (12,647 patients in 126 hospitals, 2004-2006) in Japan showed nearly 100\% 5-year overall and disease-specific survival rates if curative resection for absolute and expanded indications was performed. ${ }^{53}$ However, the role of additional surgery or endoscopic treatment should be considered in the interpretation of long-term therapeutic

Table 1. Comparison of Therapeutic Outcomes between a Meta-Analysis and Retrospective Multicenter Cohort Study

\begin{tabular}{|c|c|c|}
\hline & Meta-analysis published in $2014^{4}$ & Retrospective multicenter cohort study \\
\hline $\begin{array}{l}\text { Included studies or centers (published year } \\
\text { or duration of enrollment) }\end{array}$ & 14 studies (2009-2014) & 8 centers $(2006-2015)$ \\
\hline Enrolled cases & 972 EGC-UH & 275 EGC-UH \\
\hline $\begin{array}{l}\text { En bloc resection rate (rate of lesions that } \\
\text { met expanded criteria) }\end{array}$ & $92.1 \%(91.2 \%)$ & $92.4 \%(95.4 \%)$ \\
\hline $\begin{array}{l}\text { Complete resection rate (rate of lesions that } \\
\text { met expanded criteria) }\end{array}$ & $77.5 \%(85.6 \%)$ & $80 \%(91.7 \%)$ \\
\hline $\begin{array}{l}\text { Curative resection rate (rate of lesions that } \\
\text { met expanded criteria) }\end{array}$ & $61.4 \%(79.8 \%)$ & $36.4 \%(91.7 \%)$ \\
\hline $\begin{array}{l}\text { Recurrence rate (rate of lesions that met } \\
\text { expanded criteria in a retrospective multi- } \\
\text { center study) }\end{array}$ & $\begin{array}{l}\text { 7.6\% (95\% confidence interval: } 3.4 \%-16 \%) \\
\text { (range of follow-up duration: } 13.5-101.9 \\
\text { mo) }\end{array}$ & $\begin{array}{c}10.2 \%(9.2 \%) \text { (median follow-up with } \\
\text { interquartile range: } 3.96 \mathrm{yr} \\
(1.11-5.09 \mathrm{yr})\end{array}$ \\
\hline
\end{tabular}

En bloc resection is defined as complete removal of cancer in a single piece without fragmentation. Complete resection is defined as removal of cancer with no neoplastic components at the lateral or vertical margins and without lymphovascular invasion on microscopic examination. Curative resection is defined as removal of cancer with intramucosal lesions measuring $\leq 20 \mathrm{~mm}$ and without ulceration, neoplastic components at the lateral or vertical margins, or lymphovascular invasion. ${ }^{3,5}$

EGC-UH, early gastric cancer with undifferentiated-type histology. 
outcomes. A retrospective nationwide registry of gastric cancer $(13,626$ patients in 208 hospitals, 2002) in Japan showed a $75.7 \%$ 5-year overall survival rate in differentiated-type gastric cancer and a 65.5\% 5-year overall survival rate in undifferentiated-type gastric cancer. ${ }^{54}$ These patients were treated with endoscopic or surgical resection; however, it is difficult to interpret these numbers without information about whether the additional treatments were performed or not. In a Korean multicenter study including all cases of resected EGC-UH, the overall mortality was $1.8 \%$ and this value was lower in lesions with curative resection (1\%, median follow-up 3.96 years) and there was no cancer-related death in a cohort. ${ }^{5}$ The analysis of the role of additional treatment was also impossible in this study and caution is required for the interpretation of favorable long-term outcomes.

The interpretation of recurrence is also difficult. Recurrence was the most common cause of death in patients who underwent surgery due to advanced gastric cancer in a retrospective nationwide registry of gastric cancer in Japan. ${ }^{54}$ However, peritoneal metastasis was most frequent and the impact of local recurrence or metachronous intragastric recurrence was not differentiated. Moreover, it is not known whether the mode of metastasis including hematogenous, lymphoid, or disseminated spread should be discriminated. ${ }^{55}$

Moreover, when endoscopists choose therapeutic options for EGC, they always select optimal candidates for ESD. Baseline heath status, comorbidity, and patient preference are also important considerations. The 5-year survival rate after surgical gastrectomy is lower in patients aged $\geq 75$ years than in young or middle-aged patients, and ESD is initially recommended when it is difficult to determine the depth of invasion. $^{37,56}$

Selection bias, whether or not additional treatment after ESD was done, and whether therapeutic outcomes could represent the short-term and long-term prognosis in each study should be considered in the interpretation of the results.

\section{CONCLUSIONS}

More ways are needed to obtain an accurate initial endoscopic diagnosis to overcome histologic discrepancy between pre-ESD- and post-ESD diagnoses. ESD for EGC-MH (differentiated-type combined with undifferentiated-type gastric cancer), for a single category (EGC-UH) of PDC and SRC or for GCLS, requires more data. In addition, subdivision of indications and validation is still necessary to lessen the limitations of current ESD indications according to the histology of EGC. More precise adaptation of ESD indications is warranted and studies including all cases of resected EGC-UH regardless of the indication or criteria reflect actual experience. Selection bias, whether additional treatment after ESD was done, and whether the therapeutic outcomes could represent the shortterm and long-term prognosis in each study should be considered in the interpretation of the results.

\section{Conflicts of Interest}

The authors have no financial conflicts of interest.

\section{Author Contributions}

Conceptualization: Chang Seok Bang, Gwang Ho Baik

Resources: CSB, GHB

Supervision: CSB

Writing-original draft: CSB

Writing-review\&editing: CSB, GHB

\section{REFERENCES}

1. Gotoda T. Endoscopic resection of early gastric cancer. Gastric Cancer 2007;10:1-11.

2. Soetikno R, Kaltenbach T, Yeh R, Gotoda T. Endoscopic mucosal resection for early cancers of the upper gastrointestinal tract. J Clin Oncol 2005;23:4490-4498.

3. Gotoda T, Yanagisawa A, Sasako M, et al. Incidence of lymph node metastasis from early gastric cancer: estimation with a large number of cases at two large centers. Gastric Cancer 2000;3:219-225.

4. Bang CS, Baik GH, Shin IS, et al. Endoscopic submucosal dissection for early gastric cancer with undifferentiated-type histology: a meta-analysis. World J Gastroenterol 2015;21:6032-6043.

5. Bang CS, Park JM, Baik GH, et al. Therapeutic outcomes of endoscopic resection of early gastric cancer with undifferentiated-type histology: a Korean ESD registry database analysis. Clin Endosc 2017;50:569-577.

6. Lee JH, Kim JG, Jung HK, et al. Clinical practice guidelines for gastric cancer in Korea: an evidence-based approach. J Gastric Cancer 2014;14:87-104

7. Japanese Gastric Cancer Association. Japanese gastric cancer treatment guidelines 2010 (ver. 3). Gastric Cancer 2011;14:113-123.

8. Ono H, Yao K, Fujishiro M, et al. Guidelines for endoscopic submucosal dissection and endoscopic mucosal resection for early gastric cancer. Dig Endosc 2016;28:3-15.

9. Lee HH, Song KY, Park CH, Jeon HM. Undifferentiated-type gastric adenocarcinoma: prognostic impact of three histological types. World J Surg Oncol 2012;10:254.

10. Japanese Gastric Cancer Association. Japanese gastric cancer treatment guidelines 2014 (ver. 4). Gastric Cancer 2017;20:1-19.

11. Watanabe H, Jass JR, Sobin LH. Histological typing of oesophageal and gastric tumours: in collaboration with pathologists in 8 countries. Berlin: Springer Science \& Business Media; 2012.

12. Japanese Gastric Cancer Association. Japanese classification of gastric carcinoma - 2nd English edition. Gastric Cancer 1998;1:10-24.

13. Lee JH, Min YW, Lee JH, et al. Diagnostic group classifications of gastric neoplasms by endoscopic resection criteria before and after treatment: real-world experience. Surg Endosc 2016;30:3987-3993.

14. Min BH, Kang KJ, Lee JH, et al. Endoscopic resection for undifferentiated early gastric cancer: focusing on histologic discrepancies between forceps biopsy-based and endoscopic resection specimen-based diagnosis. Dig Dis Sci 2014;59:2536-2543.

15. Lee JH, Kim JH, Rhee K, et al. Undifferentiated early gastric cancer diagnosed as differentiated histology based on forceps biopsy. Pathol Res Pract 2013;209:314-318 
16. Kim JH. Important considerations when contemplating endoscopic resection of undifferentiated-type early gastric cancer. World J Gastroenterol 2016;22:1172-1178.

17. Yamamoto Y, Fujisaki J, Hirasawa T, et al. Therapeutic outcomes of endoscopic submucosal dissection of undifferentiated-type intramucosal gastric cancer without ulceration and preoperatively diagnosed as 20 millimetres or less in diameter. Dig Endosc 2010;22:112-118.

18. Abe S, Oda I, Suzuki H, et al. Short- and long-term outcomes of endoscopic submucosal dissection for undifferentiated early gastric cancer. Endoscopy 2013;45:703-707.

19. Mikami K, Hirano Y, Futami K, Maekawa T. Expansion of lymph node metastasis in mixed-type submucosal invasive gastric cancer. Asian J Surg 2018;41:462-466.

20. Isomoto H. Differentiated-type predominant mixed-histology-type early gastric cancer is a significant risk factor for endoscopic non-curative resection regardless of tumor size. Dig Endosc 2018;30:602-604.

21. Iwamoto J, Mizokami Y, Ito M, et al. Clinicopathological features of undifferentiated mixed type early gastric cancer treated with endoscopic submucosal dissection. Hepatogastroenterology 2010;57:185-190.

22. Zheng HC, Li XH, Hara T, et al. Mixed-type gastric carcinomas exhibit more aggressive features and indicate the histogenesis of carcinomas. Virchows Arch 2008;452:525-534.

23. Park SY, Kook MC, Kim YW, Cho NY, Kim TY, Kang GH. Mixed-type gastric cancer and its association with high-frequency CpG island hypermethylation. Virchows Arch 2010;456:625-633.

24. Saito A, Shimoda T, Nakanishi Y, Ochiai A, Toda G. Histologic heterogeneity and mucin phenotypic expression in early gastric cancer. Pathol Int 2001;51:165-171.

25. Sekiguchi M, Sekine S, Oda I, et al. Risk factors for lymphatic and venous involvement in endoscopically resected gastric cancer. J Gastroenterol 2013;48:706-712.

26. Takizawa K, Ono H, Kakushima N, et al. Risk of lymph node metastases from intramucosal gastric cancer in relation to histological types: how to manage the mixed histological type for endoscopic submucosal dissection. Gastric Cancer 2013;16:531-536.

27. Yoon HJ, Kim YH, Kim JH, et al. Are new criteria for mixed histology necessary for endoscopic resection in early gastric cancer? Pathol Res Pract 2016;212:410-414.

28. Min BH, Kim KM, Park CK, et al. Outcomes of endoscopic submucosal dissection for differentiated-type early gastric cancer with histological heterogeneity. Gastric Cancer 2015;18:618-626.

29. Horiuchi Y, Fujisaki J, Yamamoto N, et al. Undifferentiated-type component mixed with differentiated-type early gastric cancer is a significant risk factor for endoscopic non-curative resection. Dig Endosc 2018;30:624-632.

30. Lee HJ, Kim GH, Park DY, et al. Is endoscopic submucosal dissection safe for papillary adenocarcinoma of the stomach? World J Gastroenterol 2015;21:3944-3952.

31. Lee HJ, Kim GH, Park DY, et al. Endoscopic submucosal dissection for papillary adenocarcinoma of the stomach: is it really safe? Gastric Cancer 2017;20:978-986.

32. Min BH, Byeon SJ, Lee JH, et al. Lymphovascular invasion and lymph node metastasis rates in papillary adenocarcinoma of the stomach: implications for endoscopic resection. Gastric Cancer 2018;21:680-688.

33. Adachi Y, Yasuda K, Inomata M, Sato K, Shiraishi N, Kitano S. Pathology and prognosis of gastric carcinoma: well versus poorly differentiated type. Cancer 2000;89:1418-1424.

34. Hyung WJ, Noh SH, Lee JH, et al. Early gastric carcinoma with signet ring cell histology. Cancer 2002;94:78-83.

35. Kim H, Kim JH, Lee YC, et al. Growth patterns of signet ring cell carcinoma of the stomach for endoscopic resection. Gut Liver 2015;9:720726.

36. Lee IS, Lee S, Park YS, Gong CS, Yook JH, Kim BS. Applicability of endoscopic submucosal dissection for undifferentiated early gastric cancer: mixed histology of poorly differentiated adenocarcinoma and signet ring cell carcinoma is a worse predictive factor of nodal metastasis. Surg Oncol 2017;26:8-12.

37. Horiuchi Y, Fujisaki J, Yamamoto N, et al. Mixed poorly differentiated adenocarcinoma in undifferentiated-type early gastric cancer predicts endoscopic noncurative resection. Gastric Cancer 2018;21:689-695.

38. Watanabe H, Enjoji M, Imai T. Gastric carcinoma with lymphoid stroma. Its morphologic characteristics and prognostic correlations. Cancer 1976;38:232-243.

39. Nakamura S, Ueki T, Yao T, Ueyama T, Tsuneyoshi M. Epstein-Barr virus in gastric carcinoma with lymphoid stroma. Special reference to its detection by the polymerase chain reaction and in situ hybridization in 99 tumors, including a morphologic analysis. Cancer 1994;73:22392249.

40. Corvalan A, Ding S, Koriyama C, et al. Association of a distinctive strain of Epstein-Barr virus with gastric cancer. Int J Cancer 2006;118:17361742.

41. Takada K. Epstein-Barr virus and gastric carcinoma. Mol Pathol 2000;53:255-261.

42. Lim H, Lee IS, Lee JH, et al. Clinical application of early gastric carcinoma with lymphoid stroma based on lymph node metastasis status. Gastric Cancer 2017;20:793-801.

43. Lim H, Park YS, Lee JH, et al. Features of gastric carcinoma with lymphoid stroma associated with Epstein-Barr virus. Clin Gastroenterol Hepatol 2015;13:1738-1744.e2.

44. Grogg KL, Lohse CM, Pankratz VS, Halling KC, Smyrk TC. Lymphocyte-rich gastric cancer: associations with Epstein-Barr virus, microsatellite instability, histology, and survival. Mod Pathol 2003;16:641-651.

45. Lee JH, Kim SH, Han SH, An JS, Lee ES, Kim YS. Clinicopathological and molecular characteristics of Epstein-Barr virus-associated gastric carcinoma: a meta-analysis. J Gastroenterol Hepatol 2009;24:354-365.

46. Kim GH. Endoscopic submucosal dissection for early gastric cancers with uncommon histology. Clin Endosc 2016;49:434-437.

47. Lee HE, Chae SW, Lee YJ, et al. Prognostic implications of type and density of tumour-infiltrating lymphocytes in gastric cancer. Br J Cancer 2008;99:1704-1711.

48. Shin DH, Kim GH, Lee BE, et al. Clinicopathologic features of early gastric carcinoma with lymphoid stroma and feasibility of endoscopic submucosal dissection. Surg Endosc 2017;31:4156-4164.

49. Kim JH, Kim YH, Jung DH, et al. Follow-up outcomes of endoscopic resection for early gastric cancer with undifferentiated histology. Surg Endosc 2014;28:2627-2633.

50. Yamaguchi N, Isomoto H, Fukuda E, et al. Clinical outcomes of endoscopic submucosal dissection for early gastric cancer by indication criteria. Digestion 2009;80:173-181.

51. Okada K, Fujisaki J, Yoshida T, et al. Long-term outcomes of endoscopic submucosal dissection for undifferentiated-type early gastric cancer. Endoscopy 2012;44:122-127.

52. Park CH, Shin S, Park JC, et al. Long-term outcome of early gastric cancer after endoscopic submucosal dissection: expanded indication is comparable to absolute indication. Dig Liver Dis 2013;45:651-656.

53. Tanabe S, Hirabayashi S, Oda I, et al. Gastric cancer treated by endoscopic submucosal dissection or endoscopic mucosal resection in Japan from 2004 through 2006: JGCA nationwide registry conducted in 2013. Gastric Cancer 2017;20:834-842.

54. Nashimoto A, Akazawa K, Isobe Y, et al. Gastric cancer treated in 2002 in Japan: 2009 annual report of the JGCA nationwide registry. Gastric Cancer 2013;16:1-27.

55. Pernot S, Voron T, Perkins G, Lagorce-Pages C, Berger A, Taieb J. Signet-ring cell carcinoma of the stomach: impact on prognosis and specific therapeutic challenge. World J Gastroenterol 2015;21:11428-11438.

56. Kusano C, Iwasaki M, Kaltenbach T, Conlin A, Oda I, Gotoda T. Should elderly patients undergo additional surgery after non-curative endoscopic resection for early gastric cancer? Long-term comparative outcomes. Am J Gastroenterol 2011;106:1064-1069. 